\title{
THE TRUNCATED EXPONENTIAL POLYNOMIALS, THE ASSOCIATED HERMITE FORMS AND APPLICATIONS
}

\author{
G. DATTOLI AND M. MIGLIORATI
}

Received 3 April 2006; Accepted 3 April 2006

We discuss the properties of the truncated exponential polynomials and develop the theory of new form of Hermite polynomials, which can be constructed using the truncated exponential as a generating function. We derive their explicit forms and comment on their usefulness in applications, with particular reference to the theory of flattened beams, used in optics.

Copyright (c) 2006 Hindawi Publishing Corporation. All rights reserved.

\section{Introduction}

In a previous paper, Dattoli et al. [5] have discussed the properties of families of Hermite polynomials, defined through the generating function (g.f.)

$$
\sum_{n=0}^{\infty} \frac{t^{n}}{n !} \Phi_{n}(x, y)=f\left(x t+y t^{2}\right) .
$$

The function $f(x)$, called the support function, is assumed to be infinitely differentiable, to admit the series expansion

$$
f(x)=\sum_{r=0}^{\infty} \frac{f_{r}}{r !} x^{r}
$$

and the "semigroup" property

$$
f(x+y)=\hat{f}(y) f(x)
$$

The operator function $\hat{f}(y)$ is defined as

$$
\widehat{f}(y)=\sum_{r=0}^{\infty} \frac{\hat{f}_{+}^{r}}{r !} y^{r}
$$

Hindawi Publishing Corporation

International Journal of Mathematics and Mathematical Sciences

Volume 2006, Article ID 98175, Pages 1-10

DOI 10.1155/IJMMS/2006/98175 
2 Truncated exponential polynomials

with $\hat{f}_{+}^{r}$ acting on $f_{s}$ in such a way that

$$
\hat{f}_{+}^{r} f_{s}=f_{s+r} .
$$

Under these assumptions, the polynomials $\Phi_{n}(x, y)$ can explicitly be written as

$$
\Phi_{n}(x, y)=n ! \sum_{r=0}^{[n / 2]} \frac{f_{n-r} x^{n-2 r} y^{r}}{(n-2 r) ! r !} .
$$

In this paper, we will discuss a particular case of the polynomials (1.3), having as support the polynomials of the truncated exponential (TEP), namely,

$$
\sum_{n=0}^{\infty} \frac{t^{n}}{n !} H_{n}(x, y \mid m)=e_{m}\left(x t+y t^{2}\right),
$$

where

$$
e_{m}(x)=\sum_{r=0}^{m} \frac{x^{r}}{r !}
$$

are the PTE, according to the definition of [1]. The reason of the interest for this family of polynomials stems from the fact that they currently appear in the theory of the so-called flattened beams, which plays a role of paramount importance in optics and in particular in the case of super-Gaussian optical resonators (see, e.g., [6]).

A comprehensive theory of the TEP has been developed in [4], where different generalizations have been proposed and we will take some advantages from that general treatment.

The PTE can also be defined by means of the g.f.

$$
\sum_{n=0}^{\infty} t^{n} e_{n}(x)=\frac{\exp (x t)}{1-t}, \quad|t|<1,
$$

satisfying the recurrences

$$
\begin{gathered}
\frac{d}{d x} e_{n}(x)=e_{n-1}(x) \\
e_{n+1}(x)=\left[1+\frac{x}{n+1}\left(1-\frac{d}{d x}\right)\right] e_{n}(x)
\end{gathered}
$$

which can be combined to obtain the relevant second-order differential equation [1]

$$
x e_{n}^{\prime \prime}-(n+x) e_{n}^{\prime}+n e_{n}=0 .
$$

For future convenience, we will write the PTE in the form

$$
e_{n}(x)=\sum_{r=0}^{\infty} \frac{\theta(n-r)}{r !} x^{r},
$$


where $\theta(x)$ denotes the Heaviside step function, defined in such a way that

$$
\theta(x)= \begin{cases}1, & x \geq 0 \\ 0, & x<0\end{cases}
$$

By comparing (1.2) and (1.8), it is evident that in the case of PET $f_{r}=\theta(n-r)$. We can therefore obtain the explicit form of the Hermite polynomials of the truncated exponential (THP), as it will be shown in the forthcoming section.

\section{Hermite polynomials of the truncated exponential}

According to the previous discussion, the THP can explicitly be written as

$$
H_{n}(x, y \mid m)=n ! \sum_{r=0}^{[n / 2]} \frac{\theta(m-(n-r)) x^{n-2 r} y^{r}}{(n-2 r) ! r !}
$$

and we can use (1.5) and (1.6) to establish the relevant recurrences. By keeping the derivatives of both sides of (1.5) with respect to $x, y$, $t$, we obtain three recurrences, involving the continuous variables and the discrete indices, namely,

$$
\begin{gathered}
\frac{\partial}{\partial x} H_{n}(x, y \mid m)=n H_{n-1}(x, y \mid m-1), \\
\frac{\partial}{\partial y} H_{n}(x, y \mid m)=n(n-1) H_{n-2}(x, y \mid m-1), \\
H_{n+1}(x, y \mid m)=x H_{n}(x, y \mid m-1)+2 y n H_{n-1}(x, y m-1) .
\end{gathered}
$$

We can combine the first two recurrences to infer that the HPTE satisfy the "heat" type equation

$$
\begin{aligned}
\frac{\partial}{\partial y} F(x, y) & =\hat{f}_{+} \frac{\partial^{2}}{\partial x^{2}} F(x, y), \\
F(x, 0) & =\theta(m-n) x^{n}
\end{aligned}
$$

where $\hat{f}_{+}$is an operator, defined in such a way that

$$
\hat{f}_{ \pm}^{r} \theta(m)=\theta(m \pm r)
$$

(A kind of ambiguity seems to arise in the definition of the operators $\hat{f}_{ \pm}$. Strictly speaking, according to the definition given in the previous section, we should have $\bar{f}_{ \pm}^{\prime} r \theta(m-n)=$ $\theta(m-(n \pm r))$ and the operator should therefore act on the indices where the summation series is running. We prefer, for practical reasons, the definition given in (2.4), which induces discrete variations on the index $m$; it is however evident that $\bar{f}_{ \pm}^{\prime}=\bar{f}_{\mp}$.) We can handle (2.3) to obtain the following operational definition for the TEP: a proper combination of the last recurrence in (2.2) with the first yielding the identity

$$
\begin{gathered}
H_{n+1}(x, y \mid m)=\hat{M}_{m} H_{n}(x, y \mid m), \\
\hat{M}_{m}=x \hat{f}_{-}+2 y \frac{\partial}{\partial x},
\end{gathered}
$$


which, together with the first recurrence of (2.2), leads to the following second-order differential equation (see [5]):

$$
x \bar{f}_{-} \frac{\partial}{\partial x} H_{n}(x, y \mid m)+2 y \frac{\partial^{2}}{\partial x^{2}} H_{n}(x, y \mid m)-n \bar{f}_{-} H_{n}(x, y \mid m)=0 .
$$

(According to the formalism of monomiality (see [4]), we can define a multiplicative operator (in this case provided by $\widehat{M}_{m}$ ) and a derivative operator $\widehat{P}=\partial / \partial x$; the THPs are easily seen to satisfy the identity $\widehat{M}_{m} P H_{n}(x, y \mid m)=n H_{n}(x, y \mid m)$, which yields (2.6).) Needless to say, all the previous relations reduce to those of the ordinary Hermite for

$$
y=-\frac{1}{2}, \quad f_{n}=1
$$

( Note that $H_{n}(x,-1 / 2)$ can be identified with the polynomials $H e_{n}(x)$, defined by the generating function $\exp \left(x t-t^{2} / 2\right)$.) Let us now consider the problem of finding a simple formula yielding the successive derivatives, with respect to $t$, of the right-hand side of (1.5). Using the previous definitions, we get

$$
\frac{\partial^{p}}{\partial t^{p}} e_{m}\left(x t+y t^{2}\right)=\hat{H}_{p}(x+2 y t, y \mid m) e_{m}\left(x t+y t^{2}\right),
$$

where $\hat{H}(x, y \mid m)$ should be understood as an operator, defined as follows:

$$
\hat{H}_{n}(x, y \mid m)=n ! \sum_{r=0}^{[n / 2]} \frac{\hat{f}_{-}^{n-r} x^{n-2 r} y^{r}}{(n-2 r) ! r !} .
$$

The proof of the previous relations can be achieved in a fairly direct way. By multiplying, indeed, the right-hand side of (2.8) by $\xi^{p} / p$ ! and then summing over $p$, we get

$$
\begin{aligned}
\sum_{p=0}^{\infty} \frac{\xi^{p}}{p !} \frac{\partial^{p}}{\partial t^{p}} e_{m}\left(x t+y t^{2}\right) & =\exp \left(\xi \frac{\partial}{\partial t}\right) e_{m}\left(x t+y t^{2}\right) \\
& =e_{m}\left[x t+y t^{2}+(x+2 y t) \xi+\xi^{2}\right]
\end{aligned}
$$

and thus, using the semigroup property (1.2b), we easily end up with (2.8). In alternative, (2.8) can also be written as

$$
\frac{\partial^{p}}{\partial t^{p}} e_{m}\left(x t+y t^{2}\right)=p ! \sum_{r=0}^{p / 2} \frac{(x+2 y t)^{p-2 r} y^{r}}{(p-2 r) ! r !} e_{m-p-r}\left(x t+y t^{2}\right) .
$$

The results obtained in this section provide the backbone of the topics we will discuss in the forthcoming sections.

\section{Some applications of TEP and THP}

The flattened beams have been introduced in [6] to study optical systems employing the so-called super-Gaussian beams, namely, optical beams whose transverse shape is not 
reproduced by a simple Gaussian, but by a function exhibiting a quasi constant flat top as, for example,

$$
S(x, p)=\exp \left(-|x|^{p}\right), \quad p>2 .
$$

Unlike the ordinary Gaussian beams, the super-Gaussians do not have transparent propagation properties, which can easily be exploited in the design of an optical resonator.

The flattened beams defined as

$$
F(x, m)=e_{m}\left(x^{2}\right) \exp \left(-x^{2}\right)
$$

provide a good tool of approximation of a super-Gaussian, and their paraxial evolution can be treated using straightforward analytical tools.

A super-Gaussian can be reproduced according to the relation $S(x, p) \cong F(a x, m)$, with $a, m$ conveniently chosen in correspondence of the order $p$ of the super-Gaussian. An example is shown in Figure 3.1, where we have made the comparison between $F(4.76 x, 20)$ and a super-Gaussian of order $p=10$.

In Figure 3.2, we also report a comparison between a flattened beam function (FBF) and an ordinary Gaussian.

A first useful information characterizing the FBF can be obtained by deriving the differential equation they satisfy. By noting indeed that

$$
e_{m}\left(x^{2}\right)=\exp \left(x^{2}\right) F(x, m),
$$

we find from (1.7) that

$$
\begin{gathered}
\widehat{T}_{m}\left[\exp \left(x^{2}\right) F(x, m)\right]=0, \\
\widehat{T}_{m}=\xi\left(\frac{d}{d \xi}\right)^{2}-(m+\xi) \frac{d}{d \xi}+m, \\
\xi=x^{2}
\end{gathered}
$$

which explicitly yield the fairly simple form

$$
x F^{\prime \prime}(x, m)+2\left(x^{2}-m-\frac{1}{2}\right) F^{\prime}(x, m)=0
$$

The use of the rules which we have established in Section 2 allows the derivation of a simple formula yielding the successive derivatives of the FBF. By noting indeed that

$$
\left(\frac{d}{d x}\right)^{s} e_{m}\left(x^{2}\right)=\hat{H}_{s}(2 x, 1 \mid m) e_{m}\left(x^{2}\right)=s ! \sum_{r=0}^{[s / 2]} \frac{(2 x)^{s-2 r}}{(s-2 r) ! r !} e_{m-s-r}\left(x^{2}\right)
$$

and that

$$
\left(\frac{d}{d x}\right)^{s} \exp \left(-x^{2}\right)=(-1)^{s} H_{s}(2 x,-1) \exp \left(-x^{2}\right)
$$




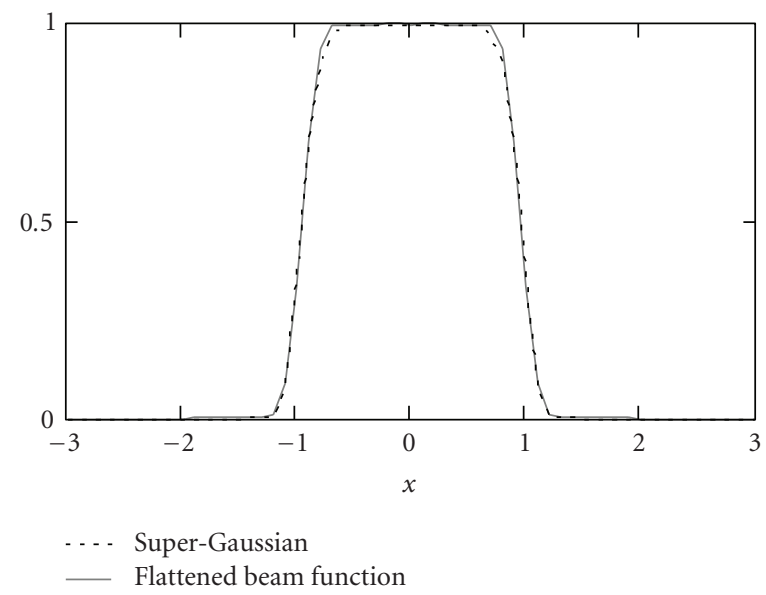

Figure 3.1. Comparison between a super-Gaussian with $p=10$ (dotted curve) and a flattened beam function $F(b x, m), b=4.76, m=20$ (solid curve); the two curves are undistinguishable.

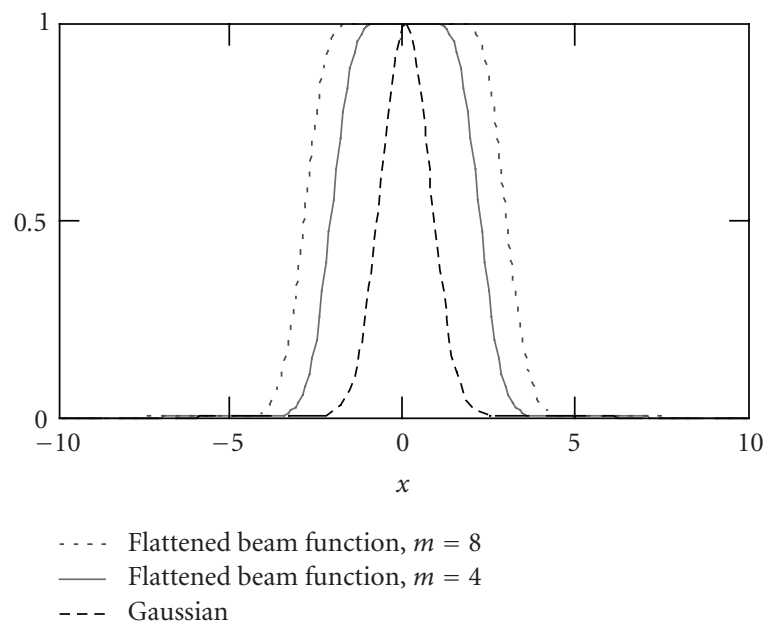

Figure 3.2. Flattened beam (dotted: $m=8$, continuous: $m=4$ ) and Gaussian (dashed).

(note that $H_{n}(2 x,-1)=H_{n}(x)=n ! \sum_{r=0}^{[n / 2]}\left((-1)^{r}(2 x)^{n-2 r} /(n-2 r) ! r !\right)$ are the ordinary Hermite defined by the g.f. $\left.\exp \left(2 x t-t^{2}\right)\right)$, we obtain

$$
\left(\frac{d}{d x}\right)^{p} F(x, m)=\sum_{r=0}^{p}\left(\begin{array}{l}
p \\
r
\end{array}\right)(-1)^{r} \hat{H}_{p-r}(2 x, 1 \mid m) H_{r}(2 x,-1) F(x, m) .
$$

Let us now discuss how the above formalism can be exploited to treat problems associated with the propagation of an FBF. 
We therefore consider the following heat-type equation:

$$
\begin{aligned}
\frac{\partial}{\partial t} Y(x, t) & =\frac{\partial^{2}}{\partial x^{2}} Y(x, t), \\
Y(x, 0) & =F(x, m),
\end{aligned}
$$

whose formal solution can be written as

$$
Y(x, t)=\exp \left(t\left(\frac{\partial}{\partial x}\right)^{2}\right) F(x, m) .
$$

To get an explicit solution, we should recall some rules of operational nature.

The use of the well-known identity

$$
\begin{aligned}
\exp \left(b \frac{\partial^{2}}{\partial x^{2}}\right)\left[x^{n} g(x)\right] & =\left[\exp \left(b \frac{\partial^{2}}{\partial x^{2}}\right) x^{n} \exp \left(-b \frac{\partial^{2}}{\partial x^{2}}\right)\right] \exp \left(b \frac{\partial^{2}}{\partial x^{2}}\right) g(x) \\
& =\left(x+2 b \frac{\partial}{\partial x}\right)^{n} \exp \left(b \frac{\partial^{2}}{\partial x^{2}}\right) g(x)
\end{aligned}
$$

yields

$$
\exp \left(t\left(\frac{\partial}{\partial x}\right)^{2}\right) F(x, m)=\sum_{r=0}^{m} \frac{(x+(2 t) \partial / \partial x)^{2 r}}{r !} \exp \left(t\left(\frac{\partial}{\partial x}\right)^{2}\right) \exp \left(-x^{2}\right)
$$

making therefore a combined use of the modified Burchnall formula (see, e.g., [3])

$$
\left(x+2 b \frac{\partial}{\partial x}\right)^{n}=\sum_{r=0}^{n}(2 b)^{r}\left(\begin{array}{l}
n \\
r
\end{array}\right) H_{n-r}(x, b)\left(\frac{\partial}{\partial x}\right)^{r}
$$

of the identity (some times referred to as the Gleisher operational rule (see [8]))

$$
\exp \left(t\left(\frac{\partial}{\partial x}\right)^{2}\right) \exp \left(-x^{2}\right)=\frac{1}{\sqrt{1+4 t}} \exp \left(-\frac{x^{2}}{1+4 t}\right)
$$

and of the following properties of the Hermite polynomials:

$$
\begin{gathered}
a^{n} H_{n}(x, y)=H_{n}\left(a x, a^{2} y\right), \\
\sum_{s=0}^{n}\left(\begin{array}{l}
n \\
s
\end{array}\right) H_{n-s}(x, y) H_{s}\left(x^{\prime}, y^{\prime}\right)=H_{n}\left(x+x^{\prime}, y+y^{\prime}\right), \\
\left(\frac{\partial}{\partial x}\right)^{n} \exp \left(a x^{2}\right)=H_{n}(2 a x, a) \exp \left(a x^{2}\right),
\end{gathered}
$$

we end up with

$$
Y(x, t)=\frac{1}{\sqrt{1+4 t}} \Omega_{m}\left(\frac{x}{1+4 t}, \frac{t}{1+4 t} \mid 2\right) \exp \left(-\frac{x^{2}}{1+4 t}\right)
$$


8 Truncated exponential polynomials

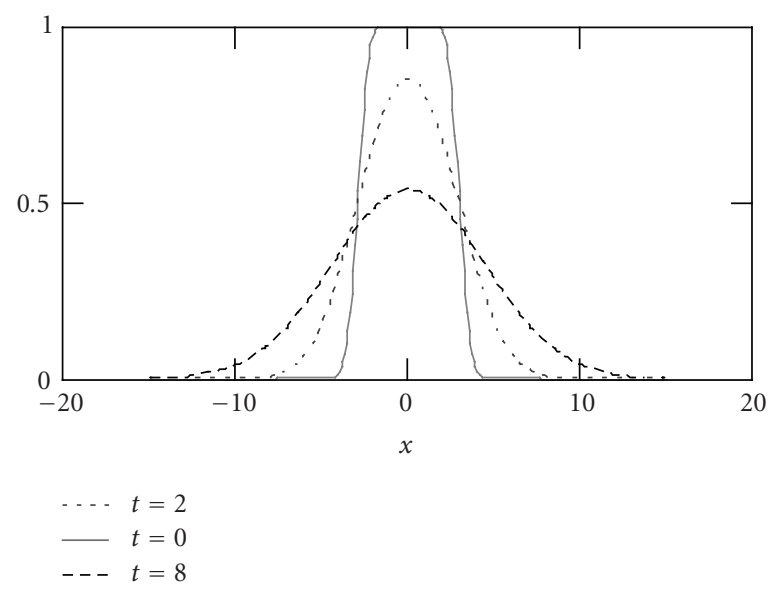

Figure 3.3. FBF evolution $m=8$ at different times (continuous: $t=0$; dotted: $t=2$; dashed: $t=8$ ).

where we have defined

$$
\Omega_{m}(x, y \mid p)=\sum_{r=0}^{m} \frac{H_{p r}(x, y)}{r !}
$$

which is a Hermite-based polynomial of the truncated exponential, whose properties have been studied in [4]. An example of evolution at different times of the $Y(x, t)$ function is given in Figure 3.3.

Analogous results can be obtained for the case of the free paraxial (Schrödinger) equation

$$
\begin{aligned}
i \frac{\partial}{\partial t} Y(x, t) & =-\frac{\partial^{2}}{\partial x^{2}} Y(x, t), \\
Y(x, 0) & =F(x, m)
\end{aligned}
$$

whose solution is omitted for the sake of conciseness.

\section{Concluding remarks}

Before closing the paper, let us add some comments aimed at better framing the obtained results.

We therefore go back to the definition of FBF and consider the evaluation of average quantities like

$$
\left\langle x^{p}\right\rangle=\int_{-\infty}^{\infty} x^{p} F(x, m) d x
$$

To perform such a calculation, we consider the following integral:

$$
\Sigma_{p, m}(a, b, c)=\int_{-\infty}^{\infty} x^{p} H_{n}(a x, b y) \exp \left(-c x^{2}\right) d x .
$$


The explicit evaluation of this integral can be done using the generating function method (1.7), thus we get

$$
\Sigma_{p, m}(a, b, c)=\sqrt{\frac{\pi}{c}} H_{m, p}\left(0, \frac{a^{2}+4 b c y}{4 c} ; 0, \frac{1}{4 c} \mid \frac{a}{2 c}\right),
$$

where

$$
H_{m, n}(x, y ; z, w \mid \zeta)=m ! n ! \sum_{r=0}^{[m, n]} \frac{\zeta^{r} H_{m-r}(x, y) H_{n-r}(z, w)}{r !(n-r) !(m-r) !}
$$

are two index Hermite polynomials (see $[2,3]$ ). It is easily checked that in the case of $(4.3)$ the odd indices are zero. It is therefore evident that the FBF root mean square at different times can be written as

$$
\left\langle x^{2}\right\rangle=\sqrt{\pi} \sum_{r=0}^{m} \frac{H_{2 r, 2}\left(0,\left(x^{2}+4 t\right) / 4(1+4 t) ; 0,(1+4 t) / 4 \mid x / 2\right)}{(r) !}
$$

which can be exploited to study the behavior of transverse section of a flattened beam.

The function

$$
g(x, m)=e_{m}\left(-x^{2}\right)
$$

can be considered a kind of truncated Gaussian and can be exploited in the theory of approximation; it shares interesting properties with the ordinary Gaussian and indeed we get

$$
(-1)^{s}\left(\frac{d}{d x}\right)^{s} e_{m}\left(-x^{2}\right)=\hat{H}_{s}(2 x,-1 \mid m) e_{m}\left(-x^{2}\right)
$$

and also the Gleisher type formula

$$
\exp \left(t\left(\frac{\partial}{\partial x}\right)^{2}\right) e_{m}\left(-x^{2}\right)={ }_{H} e_{m}\left(-x^{2}, t\right)
$$

where

$$
{ }_{H} e_{n}\left(-x^{2}, y\right)=\sum_{r=0}^{n} \frac{(-1)^{r}}{r !} H_{2 r}(x, y)
$$

We have stressed that the use of FBF simplifies the problems associated with the evaluation of beam transport, which in the case of super-Gaussian beams should be evaluated numerically. We have shown that analytical solutions can be obtained using operational methods and the properties of the TEP. Different methods can however be adopted. As also indicated in [6], the use of the expansion of the TEP in terms of Laguerre polynomials allows the use of the properties of Laguerre-Gauss optical beams, whose propagation properties are well documented in the literature (see [7]) and the method we have proposed is just an alternative to this early suggestion. 
The properties of the THPE and of their applications go far beyond the topics we have discussed in this paper. In forthcoming investigations, we will extend the present analysis and discuss the importance of THPE in combinatorics.

\section{References}

[1] L. C. Andrews, Special Functions for Engineers and Applied Mathematicians, Macmillan, New York, 1985.

[2] P. Appell and J. Kampé de Fériet, Fonctions hypérgeométriques et Hypérspheriques; polinòmes d'Hermite, Gauthier-Villars, Paris, 1926.

[3] G. Dattoli, Hermite-Bessel and Laguerre-Bessel functions: a by-product of the monomiality principle, Advanced Special Functions and Applications (Melfi, 1999) (D. Cocolicchio, G. Dattoli, and H. M. Srivastava, eds.), Proc. Melfi Sch. Adv. Top. Math. Phys., vol. 1, Aracne, Rome, 2000, pp. 147-164.

[4] G. Dattoli, C. Cesarano, and D. Sacchetti, A note on truncated polynomials, Applied Mathematics and Computation 134 (2003), no. 2-3, 595-605.

[5] G. Dattoli, H. M. Srivastava, and D. Sacchetti, The Hermite polynomials and the Bessel functions from a general point of view, International Journal of Mathematics and Mathematical Sciences 2003 (2003), no. 57, 3633-3642.

[6] F. Gori, Flattened Gaussian beams, Optics Communications 107 (1994), no. 5-6, 335-341.

[7] A. Siegman, Lasers, University Science Books, California, 1986.

[8] H. M. Srivastava and H. L. Manocha, A Treatise on Generatine Functions, Ellis-Horwood Series: Mathematics and Its Applications, John Wiley \& Sons, New York, 1984.

G. Dattoli: ENEA, UTS Tecnologie Fisiche Avanzate, Centro Ricerche Frascati,

Via Enrico Fermi 45, 00044 Frascati, Rome, Italy

E-mail address: dattoli@frascati.enea.it

M. Migliorati: Dipartimento di Energetica, Università degli Studi di Rome "La Sapienza,"

14 Via A. Scarfa, 00161 Rome, Italy

E-mail address: mauro.migliorati@uniroma1.it 


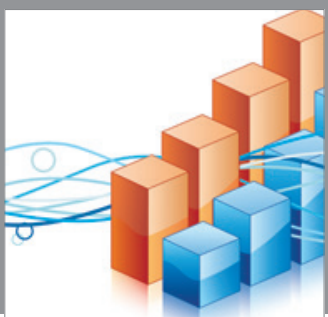

Advances in

Operations Research

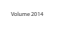

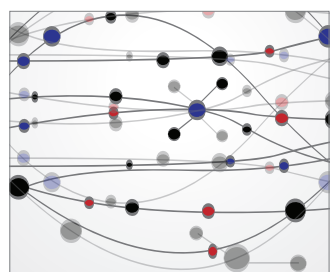

\section{The Scientific} World Journal
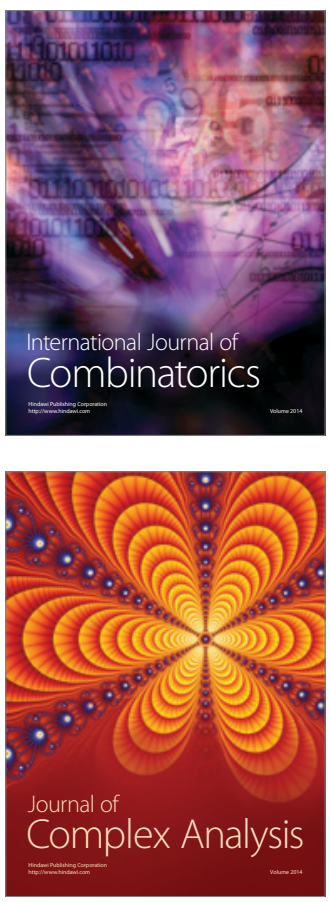

International Journal of

Mathematics and

Mathematical

Sciences
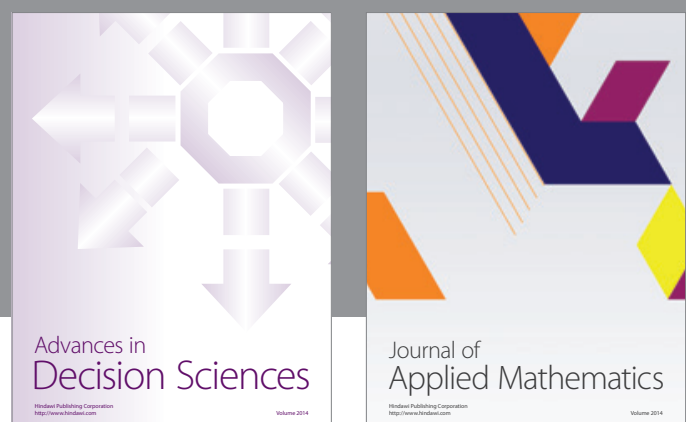

Journal of

Applied Mathematics
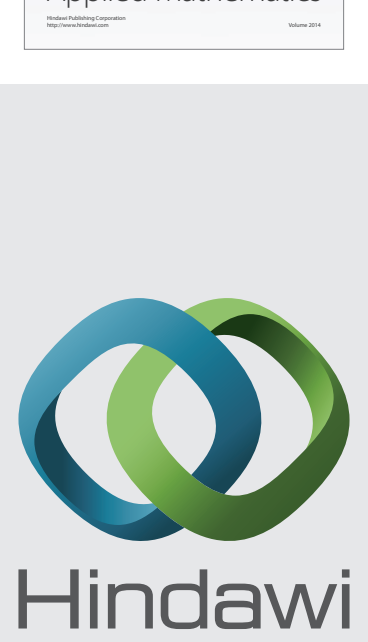

Submit your manuscripts at http://www.hindawi.com
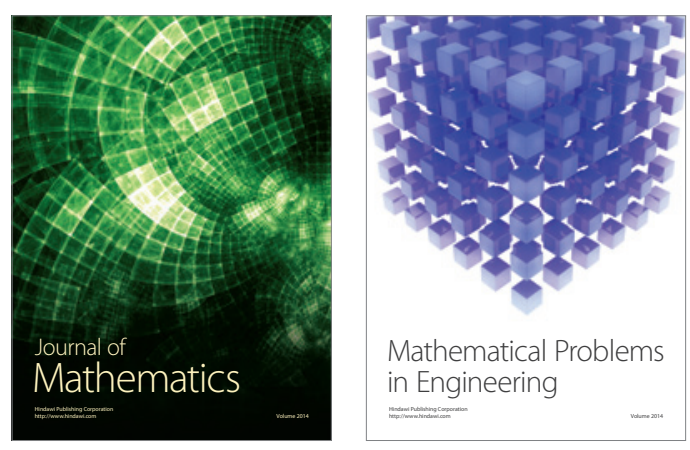

Mathematical Problems in Engineering
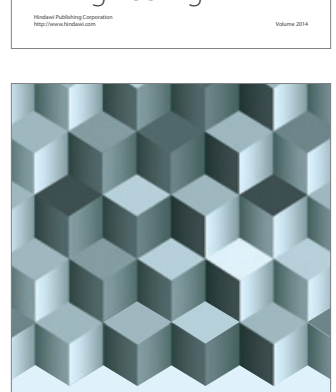

Journal of

Function Spaces
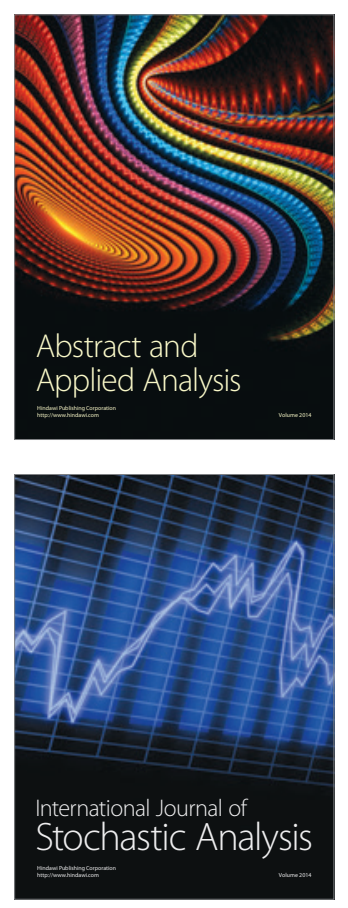

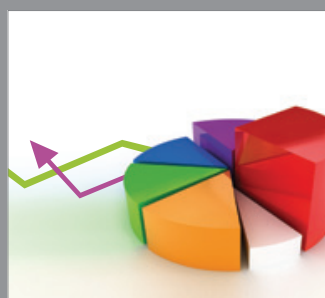

ournal of

Probability and Statistics

Promensencen
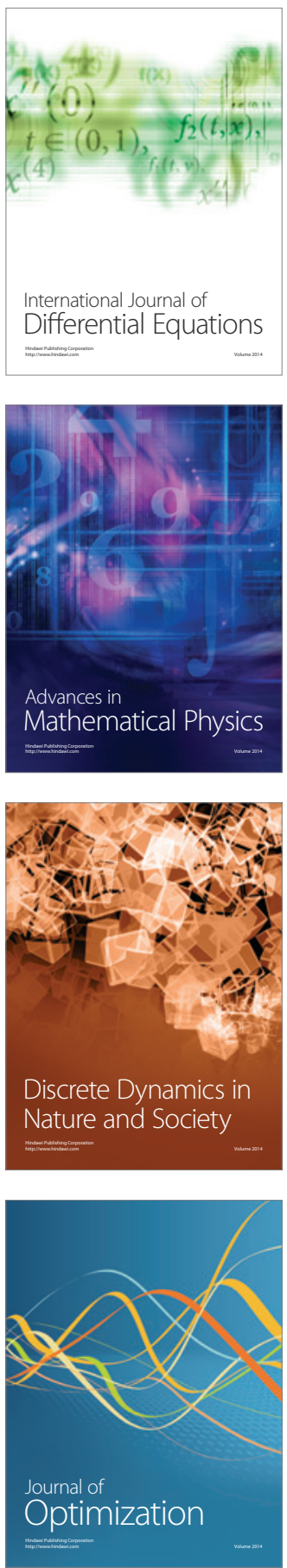